\title{
Increased co-first authorships in biomedical and clinical publications: a call for recognition
}

\author{
Marisa L. Conte, ${ }^{*}$ Stacy L. Maat, ${ }^{\dagger}$ and M. Bishr Omary ${ }^{1,+, \S}$ \\ *Taubman Health Sciences Library, ${ }^{\dagger}$ University of Michigan School of Information, ${ }^{\ddagger}$ Department of \\ Molecular and Integrative Physiology, and ${ }^{\S}$ Department of Medicine, University of Michigan Medical \\ School, Ann Arbor, Michigan, USA
}

\begin{abstract}
There has been a dramatic increase in the number and percentage of publications in biomedical and clinical journals in which two or more coauthors claim first authorship, with a change in some journals from no joint first authorship in 1990 to co-first authorship of $>30 \%$ of all research publications in 2012. As biomedical and clinical research become increasingly complex and team-driven, and given the importance attributed to first authorship by grant reviewers and promotion and tenure committees, the time is ripe for journals, bibliographic databases, and authors to highlight equal first author contributions of published original research.-Conte, M. L., Maat, S. L., Omary, M. B. Increased co-first authorships in biomedical and clinical publications: a call for recognition. FASEB J. 27, 3902-3904 (2013). www.fasebj.org
\end{abstract}

Key Words: coauthors · joint first authorship $\cdot$ citations

Publication Metrics, INCLUding the number of papers and the journals in which they are published, play an important role in the success and promotion of researchers involved in fundamental or clinical investigation at universities and research institutions $(1,2)$. The long-held convention in the biomedical and life sciences recognizes the first author (along with the corresponding or senior author) as the primary driver of the original research and resulting publication. However, our analysis of author lists in 12 major biomedical and clinical journals showed an increasing frequency of publications with joint first authors, or papers where two or more authors state that they contributed equally to the work and merit equal consideration as first authors.

Reporting of joint first authorship varies from journal to journal and can also differ on various electronic platforms (i.e., bibliographic databases or publisher websites). In the absence of a standardized format to identify authors who claim joint primary responsibility for published research, it is likely that authors whose names are not the first listed on a paper may not receive appropriate credit for their contribution. This may affect promotion, tenure, or other forms of recognition that are informed by publication metrics. To ensure appropriate recognition for equal work, we encourage journals and citation databases to begin identifying joint first authors in a way that makes it easy for readers to locate this information. In addition, establishing a convention wherein authors identify joint first authors of papers they cite in the reference section will help to reinforce the equal contributions made by researchers.

Due to a lack of standardized reporting of declaration of joint first authorship, it can be difficult or impossible for a reader of a manuscript (or its abstract in citation databases, including PubMed) to determine whether the original investigation is being published by joint first authors. Often, recognition of additional first authors becomes evident only if one refers back to the original paper and searches for the information, which can be indicated by asterisks or superscripts in the author affiliations, written in a footnote on the first page, or documented in a contribution statement at the end of the paper, to name the most common methods of reporting. In addition, citation databases or digital platforms that host published papers in HTML format often do not replicate declarations of joint authorship made in the manuscript. In other words, the HTML version of a paper on a website may not contain joint author information that is clearly stated in the PDF version of that paper available from the same website.

\section{A TREND THAT CANNOT BE IGNORED}

A review of research articles published in Gastroenterology from 2011 to 2012 reported that $21 \%$ of the manuscripts declared joint first authorship of 2 or more authors (3). To expand on the limited available data, we carried out a systematic analysis of all original research articles from 6 basic science and 6 clinically oriented journals. For both basic and clinical domains, we included 3 high-impact and 3 midlevel-impact journals, with impact factors from the 2012 Journal Citation Reports. The 6 high-impact basic and clinical journals were Cell, Science, and Nature (with impact factors of 31.96, 31.03 and 38.60, respectively) and JAMA, Lancet, and the New England Journal of Medicine (impact factors of 29.98, 39.06 and 51.66). The 6 midlevel-impact journals were FASEB Journal, Journal of Cell Science, and Oncogene (impact factors of 5.70, 5.88 and 7.36); and American Journal of Gastroenterology, Archives of Internal Medicine, and Heart (impact factors of 7.55, 10.60, and 5.01). The analysis included all

\footnotetext{
${ }^{1}$ Correspondence: Department of Molecular and Integrative Physiology, University of Michigan Medical School, 7720 Medical Science II, 1301 E. Catherine St., Ann Arbor, MI 48109-5622, USA. E-mail: mbishr@umich.edu

doi: $10.1096 /$ fj. $13-235630$
} 
research articles (full papers including systematic reviews, short reports and research letters) from each regular journal issue. Review articles, case studies, letters to the editor, and commentaries were excluded, as were all papers in supplemental issues. All volumes and issues were analyzed for 2000, 2005, 2010, and 2012; in addition, volumes and issues of basic science journals in 1990 were analyzed due to the earlier inclusion of joint first authorship declarations evidenced by preliminary data collection. To be counted as a joint first authorship, the declaration had to be clear and unambiguous. Sample statements include: "[Author A] and [Author B] contributed equally to this work and should be regarded as joint first authors," "These authors contributed equally as first authors," "These authors contributed equally to this work/study," or a superscripted character after the author's name with accompanying explanation, e.g., "Denotes co-first authorship." A total of 21,604 manuscripts were examined, and 2844 of these were the work of declared joint first authors.

The collected data show a dramatic trajectory over the last $20+$ years. In journals that publish primarily basic or translational research (Fig. $\mathbf{1} \boldsymbol{A}$ and Table 1), the percentage of papers declaring joint first authors rose sharply between 1990 and 2000 and then again between 2000 and 2012, from $0-2 \%$ in 1990 to $25-36 \%$ in 2012 . The rise nearly doubled between 2000 and 2012 and appears to be leveling off between 20-35\% (with higher percentages of joint first authored publications in higher-impact journals). Similar trends were noted for the clinically oriented journals (Fig. $1 B$ and Table 2), although the peak is lower at $8-16 \%$. The high-impact clinical journals (JAMA, Lancet, N. Engl. J. Med.), in addition to the American Jourmal of Gastroenterology, had a gradual increase between 2000 and 2012, while the numbers during the same period for Archives of Internal Medicine and Heart had either lower numbers (Arch. Intern. Med.) or a single dramatic increase during 2012 (Fig. $1 B$ and Table 2).

The publications that included joint first authors came from all parts of the globe and included numerous examples of international collaboration spanning two or more continents. Also, several other varieties of declared joint authorship were found that we did not address, including joint senior/corresponding or joint middle authors (with the former being more common than the latter). While these varieties of joint authorship may also merit standardized declaration, we believe that joint first authorship is the most important given the value of first authorship to promotion and tenure committees and to grant reviewers. Further, first authors are more likely than corresponding or senior authors to be students, postdoctoral or postprofessional degree trainees who may not yet have independent positions at
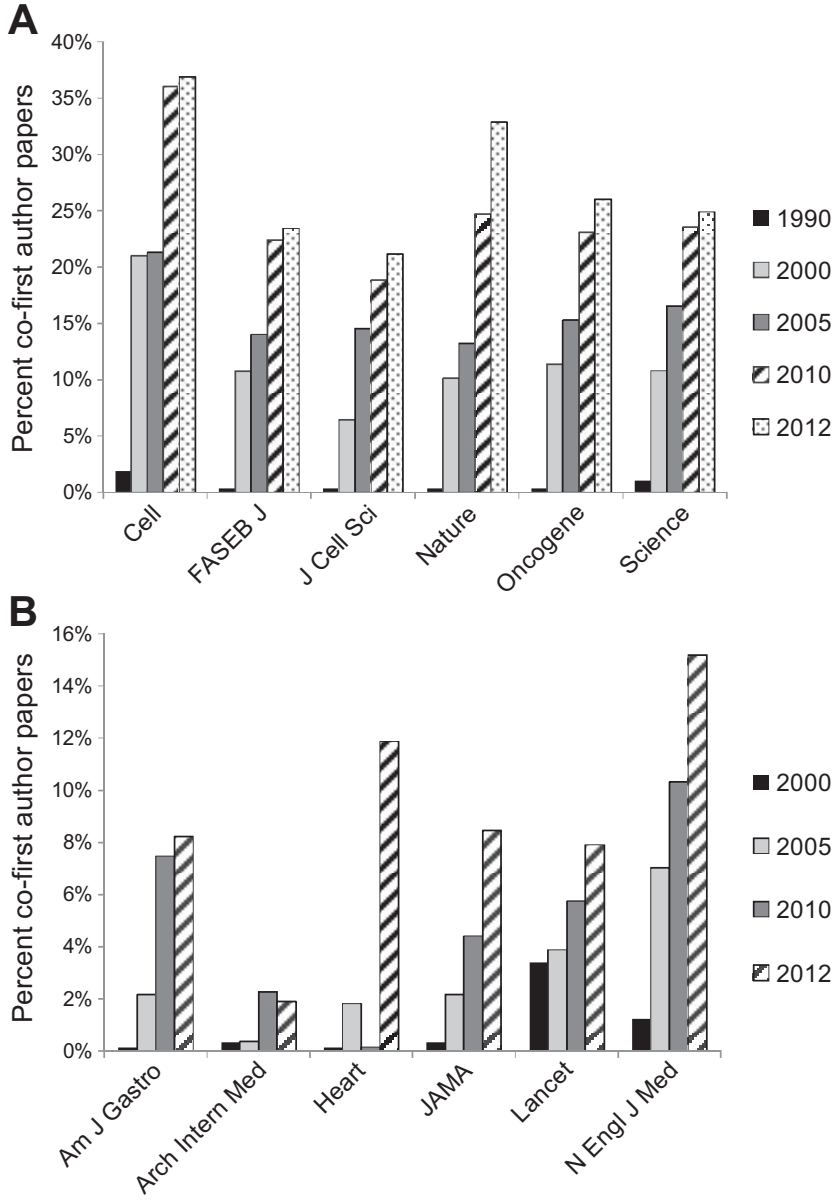

Figure 1. Trends of co-first author manuscripts in biomedical journals $(A)$ and clinical journals $(B)$.

academic or research institutions, and may therefore benefit more from the recognition that first authorship can convey.

We predict that the number of joint first author manuscripts is leveling off and will normalize between $20-40 \%$, depending on the discipline, and that the rate is unlikely to increase much beyond that range. A majority of projects will likely continue to be spearheaded by a single person (who will become the sole first author of her or his work) for a variety of reasons, including the need for investigators in training to develop independent areas of research and take ownership of specific projects. However, it is understandable that the multidisciplinary and complex nature of research nowadays easily justifies the current state of a

TABLE 1. Trends of co-first author manuscripts in biomedical journals

\begin{tabular}{|c|c|c|c|c|c|c|c|c|c|c|}
\hline \multirow[b]{2}{*}{ Journal } & \multicolumn{2}{|c|}{1990} & \multicolumn{2}{|c|}{2000} & \multicolumn{2}{|c|}{2005} & \multicolumn{2}{|c|}{2010} & \multicolumn{2}{|c|}{2012} \\
\hline & Total & Joint & Total & Joint & Total & Joint & Total & Joint & Total & Joint \\
\hline Cell & 421 & 8 & 238 & 50 & 263 & 56 & 258 & 93 & 371 & 137 \\
\hline FASEB J. & 91 & 0 & 260 & 28 & 442 & 62 & 433 & 97 & 418 & 98 \\
\hline J. Cell Sci. & 208 & 0 & 373 & 24 & 488 & 71 & 408 & 77 & 496 & 105 \\
\hline Nature & 1047 & 0 & 909 & 92 & 884 & 117 & 809 & 200 & 827 & 272 \\
\hline Oncogene & 257 & 0 & 686 & 76 & 752 & 115 & 537 & 124 & 430 & 112 \\
\hline Science & 662 & 7 & 768 & 83 & 797 & 132 & 802 & 189 & 763 & 190 \\
\hline
\end{tabular}




\begin{tabular}{|c|c|c|c|c|c|c|c|c|}
\hline \multirow[b]{2}{*}{ Journal } & \multicolumn{2}{|c|}{2000} & \multicolumn{2}{|c|}{2005} & \multicolumn{2}{|c|}{2010} & \multicolumn{2}{|c|}{2012} \\
\hline & Total & Joint & Total & Joint & Total & Joint & Total & Joint \\
\hline Am. J. Gastroenterol. & 377 & 0 & 277 & 6 & 214 & 16 & 146 & 12 \\
\hline Arch. Intern. Med. ${ }^{a}$ & 286 & 1 & 277 & 1 & 220 & 5 & 211 & 4 \\
\hline Heart & 157 & 0 & 165 & 3 & 180 & 0 & 160 & 19 \\
\hline$J A M A$ & 291 & 1 & 230 & 5 & 204 & 9 & 201 & 17 \\
\hline Lancet & 557 & 19 & 232 & 9 & 174 & 10 & 177 & 14 \\
\hline N. Engl. J. Med. & 242 & 3 & 228 & 16 & 223 & 23 & 237 & 36 \\
\hline
\end{tabular}

${ }^{a}$ Archives of Internal Medicine changed its name to JAMA Internal Medicine as of 2013.

sizable portion of published work having two or more joint first authors. It is well recognized that the number of authors on scientific papers is increasing (4) as the involvement of team science has increased $(5,6)$.

\section{A CALL FOR A COORDINATED EFFORT AMONG JOURNALS AND BIBLIOGRAPHIC DATABASES TO ACKNOWLEDGE JOINT FIRST AUTHORS}

Presently, recognition of equal contribution to original published research is often located in the fine print of a published paper or in an investigator's curriculum vitae. The analysis presented in Fig. 1 and Tables 1 and 2 raises several important points. First, the dramatic increase in joint first authors across journals covering multiple disciplines and at varying impact levels during the past 20 years indicate that co-first authorship is a trend that is likely to continue. Second, the production of a body of work that represents a publishable unit in a competitive journal often requires extensive effort by more than one lead investigator (as documented here: up to $1 / 3$ of cases in the basic/ translational-oriented journals, and up to $15 \%$ in the clinically oriented journals). This trend is likely to continue, given the increasing complexity of research and the growing reliance on team science to tackle intricate scientific and clinical problems. Third, readers of a publication of interest are most likely to recall the first author and the laboratory responsible for the work; if joint first authors are declared in some manner, then readers may be more likely to recall that more than one person made a major contribution to the study. While critics of joint first authorship may state that there can be only one "first" author and may prefer that this individual take on the extra work that justified additional joint first authors, this is increasingly unrealistic given time constraints, variability of expertise, and competition from other working groups, as well as the pressure to publish in the current challenging funding climate.

Because of the above-mentioned points and the analyses shown in Fig. 1 and Tables 1 and 2, we believe that academic journals that publish original research in the basic and clinical sciences should aim to display joint author declarations in a clear and consistent manner. Also notably, the data indicate that it is timely for journals to require authors to highlight joint first authors of papers they are citing. This can be easily done by bolding the names of joint first authors in the cited list of references. This practice has already been implemented (3), and requires authors to acknowledge joint first authorship in works cited by using bold lettering for joint first authors in the references section and appending an annotation that states: "Author names in bold designate shared co-first authorship." While some authors may perceive acknowledging joint first authorship in reference lists as tedious, this should not present major difficulties and the benefits to authors being properly acknowledged for contributions outweighs any minor inconvenience.

It is equally important that publisher platforms and databases like PubMed and PubMed Central, Google Scholar, Scopus, or Web of Science also consider steps to effect changes in reporting joint first authorship. Platforms that provide full text of articles in HTML format can begin replicating all affiliation information from the original paper, including contribution statements and declarations of joint first authorship. In addition, indicating joint first authorship in reference lists would also be helpful to readers. For example, joint first authors in a reference list could be indicated with a distinct font, color, or italics or bold lettering. This makes it easier for readers to identify papers with declared joint first authorship, and acknowledges the contributions of joint first authors.

Given the documented increase in the number of papers in the basic, clinical, and translational sciences in which multiple authors merit recognition as primary authors, acknowledgment of joint first authorship in multiple venues should be more prominent. Journal editors, publishers of bibliographic databases, and authors can all play roles to ensure that researchers receive the deserved acknowledgment for their work.

\section{REFERENCES}

1. Mavis, B., and Katz, M. (2003) Evaluation of a program supporting scholarly productivity for new investigators. Acad. Med. 78, 757-765

2. Druss, B. G., and Marcus, S. C. (2005) Tracking publication outcomes of National Institutes of Health grants. Am. J. Med. 118, 658-663

3. Dubnansky, E., and Omary, M. B. (2012) Acknowledging joint first authors of published work: the time has come. Gastroenterology 143, 879-880

4. Kennedy, D. (2003) Multiple authors, multiple problems. Science 301, 733

5. Bunton, S. A., and Mallon, W. T. (2007) The continued evolution of faculty appointment and tenure policies at U.S. medical schools. Acad. Med. 82, 281-289

6. O'Brien, T., Yamamoto, K., and Hawgood, S. (2013) Team science. Acad. Med. 88, 156-157

Received for publication May 23, 2013. Accepted for publication June 24, 2013. 